ISSN 0121-1129

elSSN 2357-5328

\title{
Evaluación de técnicas no destructivas en elementos de concreto para puentes
}

\author{
Non-destructive techniques in concrete elements for bridges' \\ evalutation \\ Avaliação de técnicas não destrutivas em elementos de \\ concreto para pontes
}

Fecha Recepción: 5 de Febrero de 2015

Fecha Aprobación: 4 de Agosto de 2015

\author{
Ricardo Alfredo-Cruz* \\ Luz Amparo Quintero-Ortiz ** \\ Carlos Andrés Galán-Pinilla*** \\ Elkin Javier Espinosa-García ${ }^{* * *}$
}

\section{Resumen}

Se evaluaron las técnicas no destructivas de Velocidad de Pulso Ultrasónico (VPU), termografía infrarroja y martillo de rebote para su aplicación en elementos de puentes de concreto y para determinar su posible aplicación in situ. El estudio se desarrolló en dos etapas: diseño y elaboración de los elementos representativos de un puente de concreto y aplicación de los ensayos no destructivos sobre estos. La técnica de VPU mostró gran capacidad para evaluar la homogeneidad y calidad de la matriz de concreto, con baja sensibilidad para identificar los daños en los refuerzos. La termografía infrarroja permitió la identificación de delaminaciones ubicadas a profundidades de hasta el 50\% del espesor de la losa evaluada. Con los resultados del martillo de rebote se estimó la resistencia a la compresión del concreto de las losas, cuyas lecturas obtenidas fueron afectadas por la presencia de agregado grueso.

Palabras clave: Velocidad de pulso ultrasónico, Termografía infrarroja, Esclerómetro, Martillo de rebote, Losas de concreto, Delaminaciones.

\footnotetext{
* Ph.D. Universidad Industrial de Santander (Bucaramanga- Santander, Colombia). racruz@uis.edu.co.

** M.Sc. Universidad Industrial de Santander (Bucaramanga- Santander, Colombia). luzquint@uis.edu.co.

*** Universidad Industrial de Santander (Bucaramanga- Santander, Colombia)

**** Universidad Industrial de Santander (Bucaramanga- Santander, Colombia)
} 


\section{Abstract}

In this work the Non-Destructive Techniques of Ultrasonic Pulse Velocity (UPV), Infrared Thermography and Rebound Hammer, used in concrete bridge elements to determine their applicability in situ, were evaluated. The study was conducted in two phases: the design and development of a concrete bridge representative elements, and their non-destructive testing application.

The UPV technique showed a great capacity to assess the concrete matrix uniformity and quality, with low sensitivity to identify the reinforcements' damages. The infrared thermography allowed to identify delaminations located at depths up to $50 \%$ of the evaluated slab thickness. With the rebound hammer results, the concrete slabs compressive strength was estimated, but their readings were affected by the coarse aggregate presence.

Keywords: Ultrasonic Pulse Velocity, Infrared Thermography, Sclerometer, Rebound Hammer, Concrete Slabs, Delaminations.

\section{Resumo}

Avaliaram-se as técnicas não destrutivas de velocidade de pulso ultrassônico (VPU), termografia infravermelha e martelo rebote para sua aplicação em elementos de pontes de concreto e para determinar sua possível aplicação in situ. O estudo desenvolveu-se em duas etapas: desenho e elaboração dos elementos representativos de uma ponte de concreto e aplicação dos ensaios não destrutivos sobre estes. A técnica de VPU mostrou grande capacidade para avaliar a homogeneidade e qualidade da matriz de concreto, com baixa sensibilidade para identificar os danos nos reforços. A termografia infravermelha permitiu a identificação de laminações localizadas a profundidades de até $50 \%$ da espessura da laje avaliada. Com os resultados do martelo rebote estimou-se a resistência à compressão do concreto das lajes, cujas leituras obtidas foram afetadas pela presença de agregado grosso.

Palavras chave: Velocidade de pulso ultrassônico, Termografia infravermelha, Esclerômetro, Martelo rebote, Lajes de concreto, Delaminações. 


\section{INTRODUCCIÓN}

Según las estadísticas, en Colombia existe un gran número de puentes; alrededor de 1668 de ellos en vías principales, y el $69 \%$ de estos son de concreto reforzado; el $24 \%$, de concreto pre-esforzado, y el $3 \%$, de acero [1]. Los puentes, en general, están sometidos a condiciones hostiles y, por lo tanto, se deterioran continuamente. Durante el período de 1986 a 2002, colapsaron en Colombia 63 puentes, 14 de ellos por deficiencias estructurales y sobrecargas [2].

Para conocer el estado de los puentes de concreto y determinar su calidad, se utilizan ensayos destructivos que, además de ocasionar interrupción de su servicio, afectan sus propiedades. Se requiere, entonces, la implementación de métodos no destructivos para la evaluación periódica de los puentes, que tengan bajo impacto sobre su funcionamiento y que permitan detectar problemas oportunamente. En este último caso se hace necesario seleccionar y determinar la confiabilidad de estas técnicas para identificar y evaluar de manera ágil y económica las zonas críticas de los elementos de concreto en puentes.

En la actualidad se han identificado la velocidad de pulso ultrasónico (VPU) [3,4], la termografía infrarroja [5] y el martillo de rebote como algunas de las técnicas con probabilidad de brindar resultados satisfactorios en la evaluación de la matriz de concreto y de los refuerzos en puentes de concreto $[6,7]$. La $V P U$ consiste en enviar un haz de ondas ultrasónicas dentro de un material y evaluar el tiempo necesario para recorrerlo; la velocidad del sonido es determinada por la relación entre el espacio recorrido y el tiempo registrado; el tiempo de vuelo del haz sonoro y, por lo tanto, la VPU son afectados por: el tipo y la calidad del concreto, la presencia de grietas o vacíos, los refuerzos de acero y su condición superficial, la humedad y la frecuencia del pulso, entre otros factores $[9,10]$. La termografia infrarroja se lleva a cabo cuando se genera un flujo de calor en el cuerpo inspeccionado utilizando estimulación de calentamiento o enfriamiento artificial o natural; la distribución de temperaturas se ve afectada por diferencias en la conducción interna de calor desde la superficie caliente, concentrándose calor en zonas discontinuas por la presencia de grietas o delaminaciones, en el caso del concreto; esta diferencia en la transferencia de calor ocurre por la variación en las propiedades térmicas del concreto y del aire presente en la interface de la grieta o delaminación [11]. El martillo de rebote es una técnica no destructiva que permite estimar la resistencia a la compresión del concreto a través del índice de rebote, que depende de la dureza superficial del hormigón sobre el cual incide, de la presencia de refuerzos, del agregado grueso subsuperficial presente y del estado de la superficie de ensayo [12, 13].

Con el fin de evaluar la sensibilidad, la rapidez y la confiabilidad de las técnicas mencionadas, se elaboraron losas de concreto con refuerzos, empleando barras con diferentes grados de daño por corrosión y torones con reducción del diámetro y fracturas de los hilos. Se evaluaron la matriz del concreto y la influencia de los daños inducidos sobre los resultados obtenidos de las técnicas.

\section{Metodología experimental}

\section{A. Diseño y elaboración de los elementos represen- tativos de un puente de concreto}

Con base en la información revisada en las referencias [12, 14-15], el manual para la inspección visual de puentes y pontones [16] y recomendaciones de personal experto en estructuras, se seleccionó la losa maciza como un elemento representativo de un puente de concreto, debido a que hace parte de la superestructura, que transmite las cargas vivas. Además, contiene material de refuerzo, como barras corrugadas y torones de acero, aspecto de interés para el estudio en esta investigación. Se utilizaron barras y torones con diámetro de $0,0125 \mathrm{~m}$ (1/2") en buen estado, y con diferentes grados de daño inducido [8,17], como corrosión (logrado mediante inmersión de las barras en solución agresiva, generando el crecimiento de una capa de óxido adherida a la superficie, inicialmente, en el $80 \%$ de la barra y, finalmente, en el $100 \%$ de la superficie de hasta $0,52 \mathrm{~mm}$ de espesor), reducciones de diámetro, fractura de hilos en el torón y fractura total. Algunas imágenes de los refuerzos y sus grados de daño se muestran en la Fig. 1. Mediante inmersión en medio agresivo se produjo el daño en las barras de refuerzo, 
generando el crecimiento de una capa de óxido de hasta $0,52 \mathrm{~mm}$.

Con base en lo anterior se diseñaron y elaboraron cuatro losas detalladas en la Tabla 1 y en la Fig. 2; en esta tabla se indican para cada losa la condición de los refuerzos y la profundidad a la cual se ubicaron.

Las losas fueron elaboradas con un diseño de mezcla para concreto de alta resistencia, siguiendo el método de la American Concrete Institute ACI 211, con relacion agua-cemento $\mathrm{a} / \mathrm{c}=0.35$, empleando aditivo plastificante reductor de agua, tamaño máximo de agregado grueso de $0,019 \mathrm{~m}$ y módulo de finura del agregado fino de 2.82. La elaboracion de la mezcla, las probetas, llenado de los moldes y curado se hizo siguiendo las recomendaciones tecnicas correspondientes.

\section{B. Aplicación de los ensayos no destructivos en los elementos}

En esta etapa se aplicaron sobre las losas de concreto las técnicas de VPU, termografía infrarroja y martillo de rebote después de 28 días de curado.

Para la aplicación de la técnica de VPU se realizó una preparación de la superficie por medio de una piedra esmeril, con el fin de tener superficies lisas y paralelas; luego se trazó una cuadrícula de $5 \mathrm{~cm}$ x $5 \mathrm{~cm}$ sobre la superficie de las zonas de los refuerzos, de modo que el haz sonoro viaja parcialmente por estas y sobre las zonas de concreto sin refuerzo, es decir, viaja sobre el concreto $\sin$ influencia de los refuerzos, como se indica en la Fig. 2. La cuadrícula trazada permitió la ubicación exacta de los transductores en las losas y hacer las respectivas mediciones.

Adicionalmente, se realizó la calibración de los equipos y la toma de las longitudes de trayectoria de la onda mediante un calibrador digital. En cada punto de evaluación se tomaron dos lecturas, cuidando de no superar el error establecido en normas técnicas, como se indica en algunas referencias [13, 14]. Con los datos obtenidos de tiempo y distancia recorrida por la onda, se calculó la velocidad de pulso. Se empleó para el estudio un equipo Pundit Plus modelo PC 1006, disponible en la UIS, y el equipo Olson Instrument NDE-360 SE-1, facilitado por una empresa de la región. Se utilizó el método directo con los refuerzos paralelos y perpendiculares a la dirección de propagación del haz sonoro, así como en zonas sin refuerzo, empleando una frecuencia de $54 \mathrm{kHz}$ y vaselina como acoplante. Los datos se tomaron haciendo un barrido sobre la losa siguiendo la cuadrícula marcada en la superficie, sobre las zonas con y sin refuerzo, tomando $0.1 \mathrm{~m}(10 \mathrm{~cm}) \mathrm{de}$ tolerancia en los bordes. 


\begin{tabular}{|c|c|}
\hline $\begin{array}{l}\text { S: } \\
\text { Diámetro } \\
\text { nominal D: } \\
12\end{array}$ & 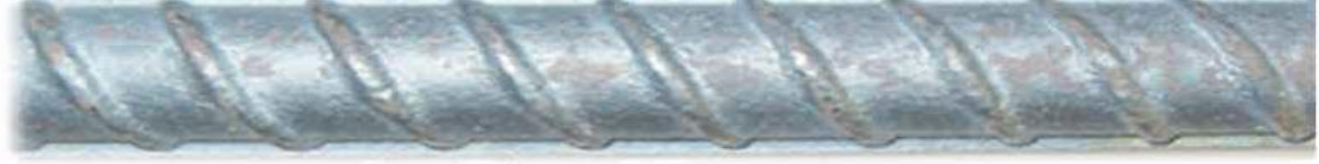 \\
\hline $\begin{array}{l}\text { CL: } \\
\text { Corrosión } \\
\text { distribuida } \\
\text { en el } 80 \% \text {, } \\
\text { D: } 12-12,5\end{array}$ & 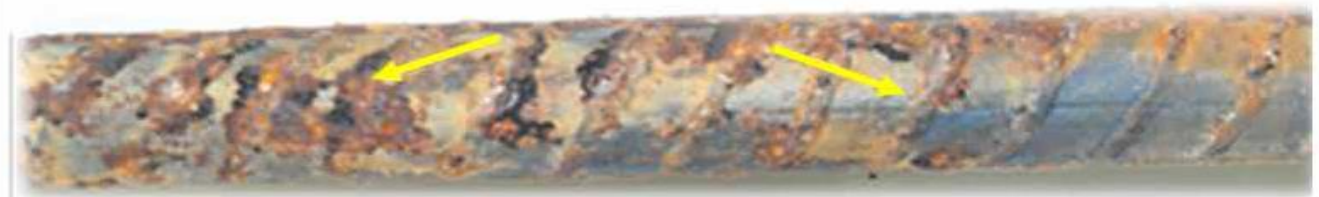 \\
\hline $\begin{array}{l}\text { CM: } \\
\text { Corrosión } \\
\text { general, D: } \\
12,5\end{array}$ & (2) \\
\hline $\begin{array}{l}\text { CS: } \\
\text { Corrosión } \\
\text { general: D: } \\
12,52\end{array}$ & 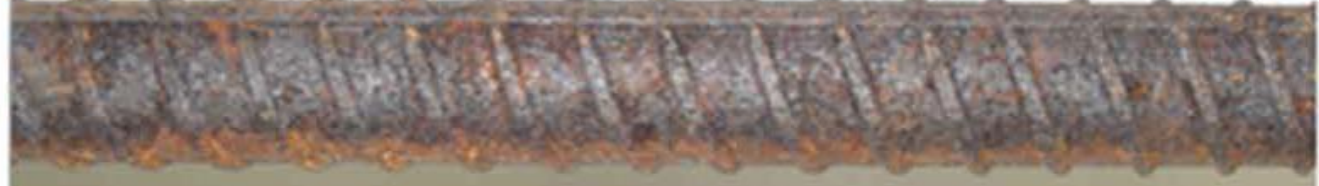 \\
\hline $\begin{array}{l}\text { R: } \\
\text { Reduccción } \\
\text { del } 60 \% \text { de } \\
\text { diámetro. }\end{array}$ & 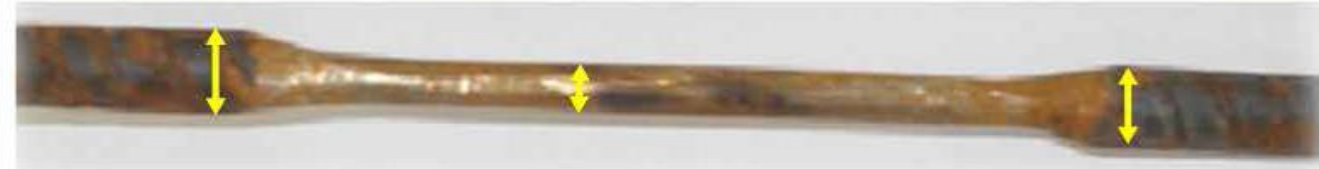 \\
\hline $\begin{array}{l}\text { S: Vista } \\
\text { frontal y } \\
\text { lateral del } \\
\text { torón sano }\end{array}$ & 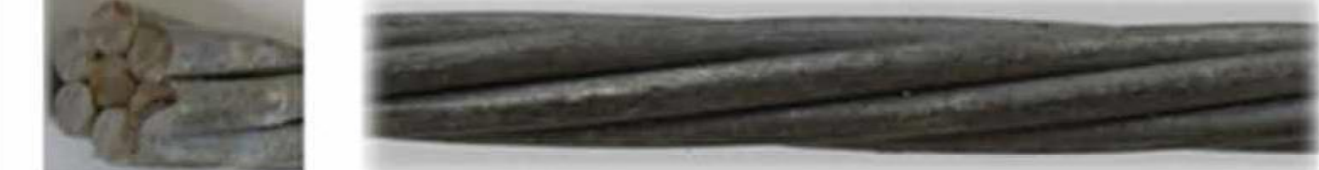 \\
\hline $\begin{array}{l}\text { T: } \\
\text { Fractura de } \\
\text { tres hilos y } \\
\text { dos hilos }\end{array}$ & 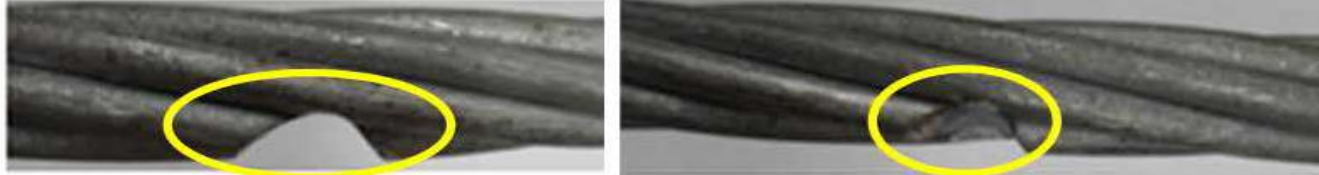 \\
\hline $\begin{array}{l}\text { R: } \\
\text { Reducción } \\
\text { del } 60 \% \text { de } \\
\text { diámetro } \\
\text { del torón }\end{array}$ & 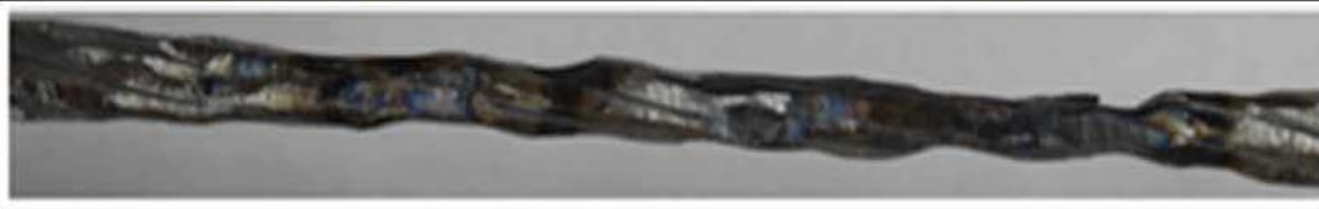 \\
\hline $\begin{array}{l}\text { F: Torón } \\
\text { fracturado y } \\
\text { barra } \\
\text { fracturada }\end{array}$ & 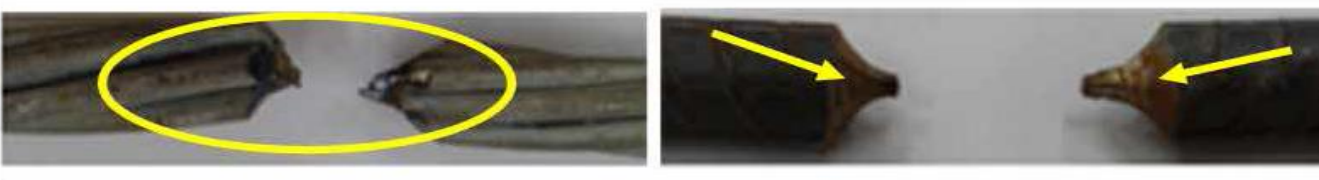 \\
\hline
\end{tabular}

Fig. 1. Imágenes mostrando grado de daño inducido en los refuerzos, S: refuerzo sano, CL: corrosión leve, CM: corrosión media, Cs: corrosión severa, R: reducciones de diámetro, T: torón con 1, 2,3 y 4 hilos fracturados, F: fractura total. 
La termografía infrarroja se aplicó a la losa 4 con el fin de identificar delaminaciones (simuladas con poliestireno) en la matriz de concreto. Se indujo calentamiento intencionalmente con exposición al sol durante un tiempo total de 5 horas, siguiendo recomendaciones técnicas indicadas en la bibliografía
$[4,9,13]$. Los termogramas fueron tomados a 3 diferentes horas: 8:00, 9:30 y 11:00 a.m. Luego de obtenidas las imágenes termográficas se procedió a realizar un análisis completo con el software Smartview suministrado por el fabricante de la cámara termográfica FLUKE.

\section{TABla 1}

CARACTERÍSTICAS DE LAS LOSAS DISEÑADAS PARA LA INVESTIGACIÓN

\begin{tabular}{|c|c|c|c|c|c|c|c|c|c|c|c|c|c|}
\hline \multicolumn{2}{|c|}{ Tipo de refuerzo } & \multirow{2}{*}{$\begin{array}{c}\text { B1 } \\
S\end{array}$} & \multirow{2}{*}{$\frac{\text { B2 }}{\mathrm{S}}$} & \multirow{2}{*}{$\frac{\text { B3 }}{S}$} & \multirow{2}{*}{$\frac{\text { B4 }}{-}$} & \multirow{2}{*}{$\begin{array}{c}\text { B5 } \\
-\end{array}$} & \multirow{2}{*}{$\begin{array}{c}\text { B6 } \\
-\end{array}$} & \multirow{2}{*}{\begin{tabular}{c|}
$\mathbf{T 1}$ \\
$\mathrm{S}$
\end{tabular}} & \multirow{2}{*}{$\frac{\mathrm{T} 2}{\mathrm{~S}}$} & \multirow{2}{*}{$\begin{array}{c}\text { T3 } \\
\mathrm{S}\end{array}$} & \multirow{2}{*}{$\begin{array}{c}\text { T4 } \\
-\end{array}$} & \multirow{2}{*}{$\begin{array}{c}\text { T5 } \\
-\end{array}$} & \multirow{2}{*}{$\begin{array}{r}\text { T6 } \\
-\end{array}$} \\
\hline Losa 1 & $\begin{array}{l}\text { Condición del } \\
\text { refuerzo }\end{array}$ & & & & & & & & & & & & \\
\hline L1 & Profundidad [cm] & 1,9 & 4,4 & 6,9 & - & - & - & 1,9 & 4,4 & 6,9 & - & - & - \\
\hline \multirow{2}{*}{$\begin{array}{l}\text { Losa } 2 \\
\text { L2 }\end{array}$} & $\begin{array}{l}\text { Condición del } \\
\text { refuerzo }\end{array}$ & $\mathrm{S}$ & $\mathrm{CL}$ & $\mathrm{CM}$ & $\mathrm{CS}$ & $\begin{array}{c}\mathrm{R} 1, \\
\mathrm{R} 2, \mathrm{R} 3 \\
\end{array}$ & $\mathrm{~F}$ & - & - & - & - & - & - \\
\hline & Profundidad [cm] & 4,4 & 4,4 & 4,4 & 4,4 & 4,4 & 4,4 & - & - & - & - & - & - \\
\hline \multirow{2}{*}{$\begin{array}{l}\text { Losa } 3 \\
\text { L3 }\end{array}$} & $\begin{array}{l}\text { Condición del } \\
\text { refuerzo }\end{array}$ & - & - & - & - & - & - & $\mathrm{S}$ & $\mathrm{T} 2, \mathrm{~T} 3, \mathrm{~T} 4$ & $\mathrm{~T} 1, \mathrm{~T} 2, \mathrm{~T} 3, \mathrm{~T} 4$ & R4 & $\mathrm{R} 3, \mathrm{R} 4, \mathrm{R} 5$ & $\mathrm{~F}$ \\
\hline & Profundidad [cm] & - & - & - & - & - & - & 4,4 & 4,4 & 4,4 & 4,4 & 4,4 & 4,4 \\
\hline \multirow{3}{*}{$\begin{array}{l}\text { Losa } 4 \\
\text { L4 }\end{array}$} & \multirow{2}{*}{$\begin{array}{l}\text { Tamaño de la } \\
\text { delaminación }[\mathrm{cm}]\end{array}$} & \multicolumn{4}{|c|}{ D1 } & \multicolumn{4}{|c|}{ D2 } & \multicolumn{4}{|c|}{ D3 } \\
\hline & & \multicolumn{4}{|c|}{$15 * 15 * 1$} & \multicolumn{4}{|c|}{$15 * 15 * 1$} & \multicolumn{4}{|c|}{$15 * 15 * 1$} \\
\hline & Profundidad $[\mathrm{cm}]$ & \multicolumn{4}{|c|}{2.5} & \multicolumn{4}{|c|}{5.0} & \multicolumn{3}{|c|}{7.5} & \\
\hline \multicolumn{14}{|c|}{ NOMENCLATURAS UTILIZADAS } \\
\hline \multicolumn{2}{|c|}{ B: Barra o varilla } & \multicolumn{2}{|c|}{ T:Torón } & \multicolumn{5}{|c|}{$\begin{array}{l}\text { R1: Reducción del } 10 \% \text { de } \\
\text { diámetro en } 10 \mathrm{~cm} \text { del refuerzo }\end{array}$} & \multicolumn{5}{|c|}{ T1: Torón con un hilo fracturado } \\
\hline \multicolumn{4}{|c|}{ S: Refuerzo sano } & \multicolumn{5}{|c|}{$\begin{array}{l}\text { R2: Reducción del } 20 \text { de } \\
\text { diámetro en } 10 \mathrm{~cm} \text { del refuerzo }\end{array}$} & \multicolumn{5}{|c|}{ T2: Torón con dos hilos fracturados } \\
\hline CL: Co & osión leve $\varnothing=13,2 \mathrm{mn}$ & & & $\begin{array}{l}\text { R3: } \mathrm{R} \\
\text { diáme }\end{array}$ & $\begin{array}{l}\text { educc } \\
\text { tro en }\end{array}$ & $\begin{array}{l}\text { ión del } 3 \\
10 \mathrm{~cm} \mathrm{~d} \\
\end{array}$ & $\begin{array}{l}\% \mathrm{~d} \\
1 \mathrm{refi}\end{array}$ & erzo & T3: Torón & con tres hilos $\mathrm{fr}$ & actura & dos & \\
\hline CM: Cc & osión media $\varnothing=13,5$ & & & $\begin{array}{l}\text { R4: } \mathrm{F} \\
\text { diáme }\end{array}$ & $\begin{array}{l}\text { educc } \\
\text { troen }\end{array}$ & $\begin{array}{l}\text { ión del } 4 \\
10 \mathrm{~cm} \mathrm{de} \\
\end{array}$ & $\begin{array}{l}\% \mathrm{~d} \\
\text { refu }\end{array}$ & & T4: Torón & con cuatro hilo & fract & rrados & \\
\hline $\begin{array}{l}\text { CS: Co } \\
\text { del refu }\end{array}$ & $\begin{array}{l}\text { sión severa } \emptyset=12,2 \text { 1 } \\
\text { zo }\end{array}$ & en 1 & $0 \mathrm{~cm}$ & $\begin{array}{l}\text { R5: } \mathrm{R} \\
\text { diáme }\end{array}$ & $\begin{array}{l}\text { educc } \\
\text { tro en }\end{array}$ & $\begin{array}{l}\text { ión de } 5 \\
10 \mathrm{~cm} \mathrm{~d}\end{array}$ & $\begin{array}{l}\% \text { de } \\
1 \text { refi }\end{array}$ & erzo & F: Fractur & & & & \\
\hline D1: Del & ninación uno & & & D2: I & elami & nación d & & & D3: Delar & inación tres & & & \\
\hline
\end{tabular}




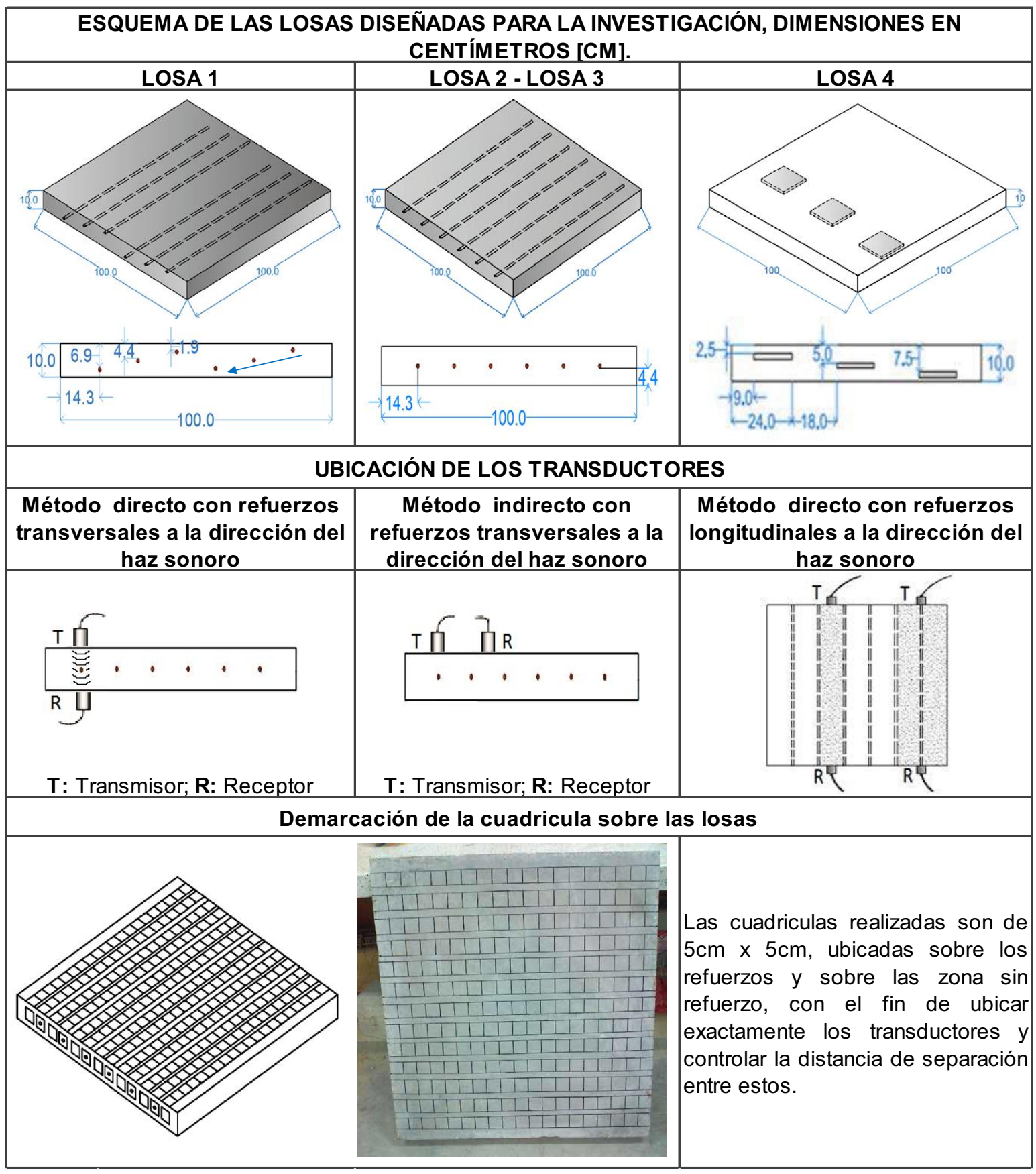

Fig. 2. Esquema de las losas diseñadas para la investigación y ubicación de los transductores; dimensiones en centímetros $[\mathrm{cm}]$.

\section{Análisis de RESUltados}

La aplicación de la VPU en las losas dos y tres, empleando el método directo a través del espesor de estas, mostró gran capacidad para evaluar la calidad del concreto, con una velocidad de pulso promedio de $3961.35 \mathrm{~m} / \mathrm{seg}$, clasificándose como un concreto de buena calidad, según los intervalos de clasificación del concreto encontrados en la bibliografía revisada $[4,9]$. La estimación de la calidad obtenida mediante la VPU fue comprobada con los resultados de las pruebas de resistencia a la compresión, en donde se obtuvo un promedio de $38.15 \mathrm{MPa}$ para probetas elaboradas con la misma mezcla de las losas. 


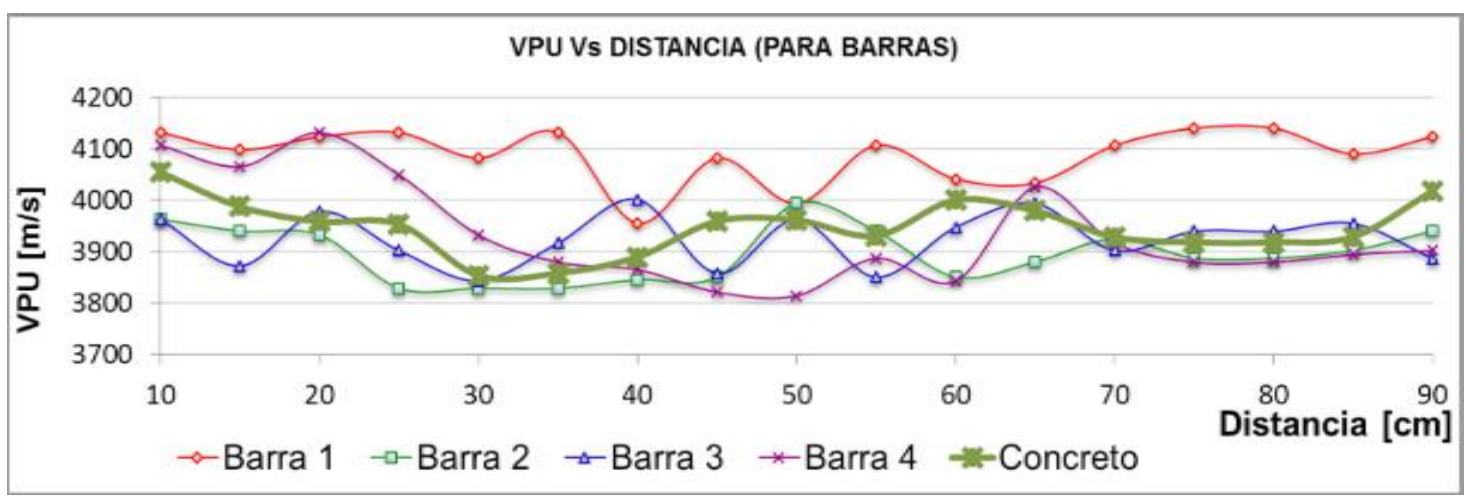

Fig. 3. Resultados de VPU sobre la losa 2 en concreto sin barras de refuerzo y con estas transversales al haz sonoro.

En la Fig. 3 se presentan los resultados de VPU medidos sobre el concreto sin refuerzos y con estos transversales a la dirección del haz sonoro, graficando el valor de velocidad obtenido en cada cuadrícula demarcada a lo largo de la losa; en la gráfica se observan los perfiles de VPU con un comportamiento heterogéneo característico del concreto y un incremento promedio de $2.45 \%$ $(141.67 \mathrm{~m} / \mathrm{s})$ para las lecturas sobre la barra en buen estado. La figura muestra una baja influencia de los refuerzos en la VPU, considerándose no relevante su presencia, coincidiendo con lo expresado por otros autores $[10,16]$. El comportamiento mostrado permite evidenciar la baja sensibilidad de la técnica para captar los refuerzos y los diferentes grados de daño presentes en estos. Lo observado se debe a la longitud de onda utilizada en el ensayo $(\lambda=0.07 \mathrm{~m})$ para la identificación de los refuerzos de diámetro $\Phi=0,0125 \mathrm{~m}(1 / 2 \mathrm{pulg})$.

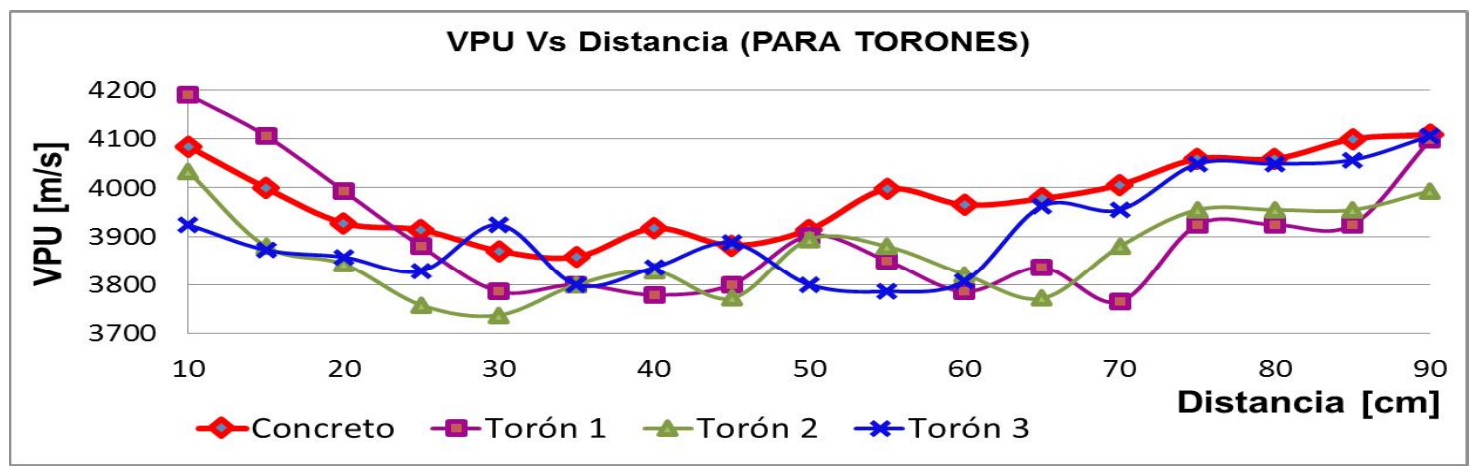

Fig. 4. Resultados de VPU sobre la losa 3 en concreto sin torones de refuerzo y con estos transversales al haz sonoro.

La Fig. 4 muestra los resultados de VPU para la losa 3 tomados de igual forma que en la losa L2. De manera similar al caso anterior, se observa un comportamiento heterogéneo característico del concreto y baja sensibilidad para la identificación de torones de $\Phi=0.0125 \mathrm{~m}(1 / 2 \mathrm{pulg})$.

Con los resultados anteriores se confirmó la capacidad de la VPU para evaluar la homogeneidad y calidad del concreto, pero no se evidenció la presencia de los refuerzos con diámetro de $\Phi=0,0125 \mathrm{~m}$ ( $1 / 2 \mathrm{pulg}$ ). Al no presentar la presencia de refuerzos influencia sobre las lecturas de VPU, se permitiría la evaluación de faltas de adherencia del refuerzo o la presencia de grietas en la matriz de concreto en la zona cercana a este.

La Fig. 5 resume las mediciones de VPU realizadas con los refuerzos longitudinales a la dirección del pulso ultrasónico para las losas L2 y L3, con presencia de barras y torones, respectivamente. Los datos se tomaron enfrentando los transductores en los extremos de los refuerzos, de modo que la onda viaja parcialmente en el concreto y parcialmente en el acero, mostrando la influencia del refuerzo y su grado de daño sobre la VPU. 


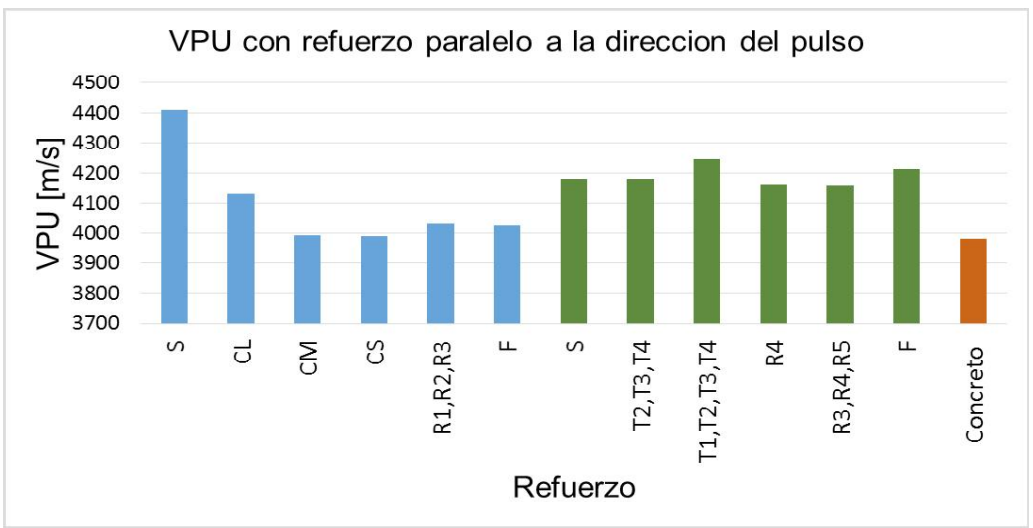

Fig. 5. Comparación de las medidas de VPU realizadas en las losas L2 y L3.

En esta figura se observa la influencia de los refuerzos en el aumento de la VPU respecto a la obtenida sobre el concreto, presentando mayor sensibilidad para diferenciar el grado de daño en las barras que en los torones. En estos últimos no fue posible diferenciar el torón sano y sus diferentes grados de daño. La VPU en la barra sana $\mathrm{S}$ alcanzó $4408.99 \mathrm{~m} / \mathrm{s}$, disminuyendo a $4133.11 \mathrm{~m} / \mathrm{s}$ en la barra con corrosión leve CL y $3993.27 \mathrm{~m} / \mathrm{s}$ la barra con corrosión media CM. Esta última presentó comportamiento similar a las barras con corrosión severa $\mathrm{CS}$, con reducción de diámetro $\mathrm{R}$ y la fracturada $\mathrm{F}$, sin que se puedan discriminar estos daños. El comportamiento anterior ocurre probablemente por el efecto que causa la capa de óxido sobre la onda, lo cual afecta la velocidad de propagación de esta.

La Fig. 6 presenta los resultados de VPU sobre la losa 1 realizados por el método indirecto con los refuerzos transversales a la dirección del pulso.

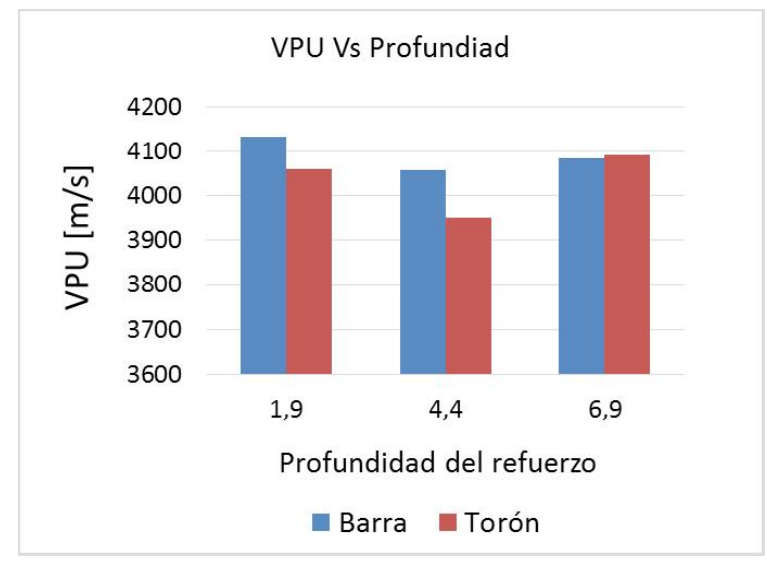

Fig. 6. Resultados de VPU medidos por el método indirecto en la losa 1.
La VPU por el método indirecto no se ve afectada por la presencia de refuerzos de $\Phi=0,0125 \mathrm{~m}(1 / 2 \mathrm{pulg})$ a profundidades de $0.019,0.044$ o $0.069 \mathrm{~m}$; este comportamiento está relacionado con la forma como se propaga la onda, siendo fuertemente afectada por el estado del concreto superficial y subsuperficial. Con base en los resultados anteriores se podría estimar la profundidad de una grieta transversal al refuerzo sin que este afecte la VPU, siguiendo las recomendaciones técnicas requeridas para la evaluación de grietas en concreto [10].

Las imágenes termográficas se tomaron siguiendo las recomendaciones de normas técnicas, a tres horas diferentes: 8:00a.m., 9:30a.m. y 11:00a.m.

En las termografías obtenidas a las 8:00a.m., Fig. 7, se observa un diferencial térmico debido a la concentración de calor en las zonas con presencia de delaminaciones que disminuyen la trasferencia de calor. La delaminación D1, a $2.5 \mathrm{~cm}$ de profundidad, presentó una temperatura de $38.6^{\circ} \mathrm{C}$; la D2, a $5 \mathrm{~cm}$ de profundidad, presentó una temperatura de $39.1^{\circ} \mathrm{C}$, y la $\mathrm{D} 3$, a $7.5 \mathrm{~cm}$ de profundidad, no presentó un aumento de temperatura respecto al concreto sano. La delaminación D2 presentó una temperatura mayor que la D1 debido a la trasferencia de calor por convección que ocurre en las caras laterales de la losa.

Las termografías obtenidas a las 9:30a.m., Fig. 8, presentan el mismo comportamiento térmico, concentrándose mayor calor en la delaminación D2; con este tiempo de exposición se logra detectar la delaminación D3 y se mejora la visibilidad de las anteriores, debido al mayor diferencial térmico entre las zonas sanas y las zonas con delaminaciones. 
a)

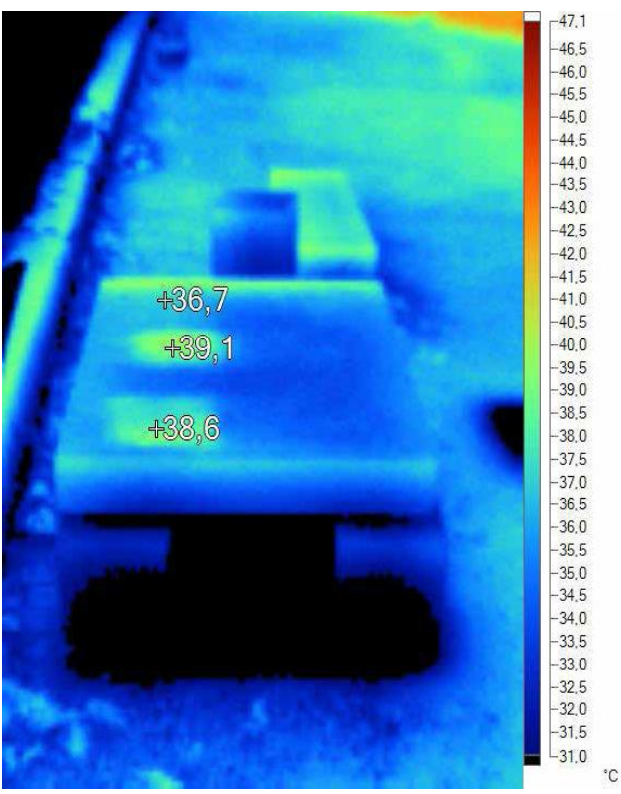

b)

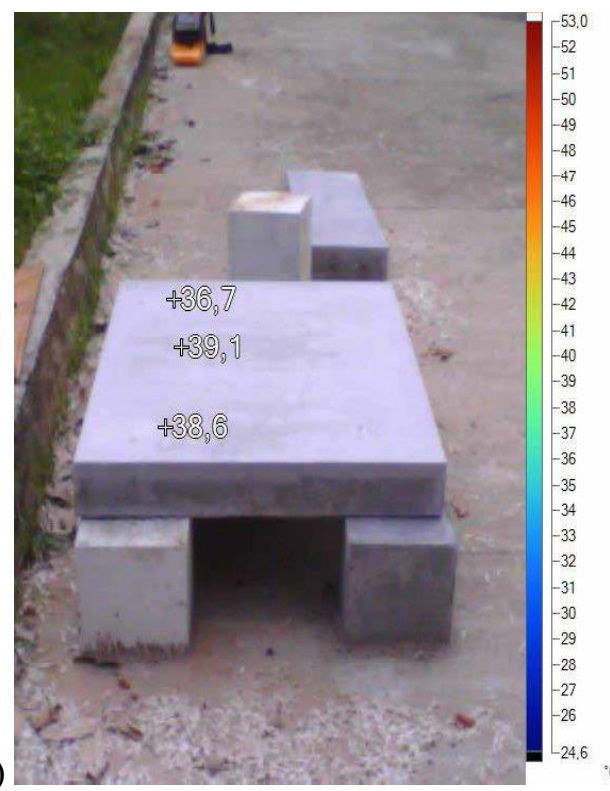

FiG. 7. a) Imagen termográfica a las 8:00 a.m., b) Imagen visible a las 8:00 a.m.

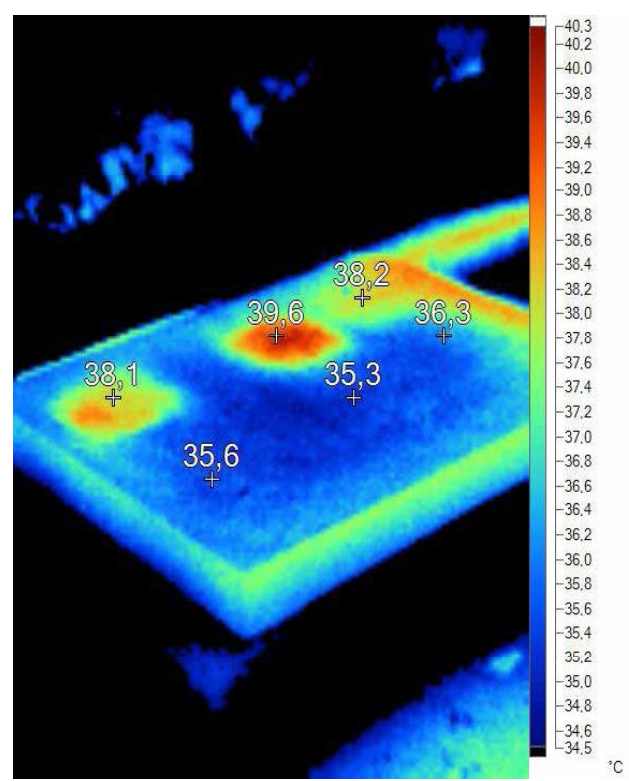

Fig. 8. a) Imagen termografía a las 9:30 a.m. b) Imagen visible a las 9:30 a.m.
Las termografías obtenidas a las 11:00a.m., Fig. 9, mantienen el comportamiento térmico con dificultad para delimitar D3, que se confunde con el borde donde se concentra calor, debido a que es sitio de incidencia del Sol.
Los resultados muestran gran capacidad de esta técnica para detectar las delaminaciones simuladas, convirtiéndose en una alternativa para la inspección de elementos de concreto que estén expuestos a la radiación solar. 

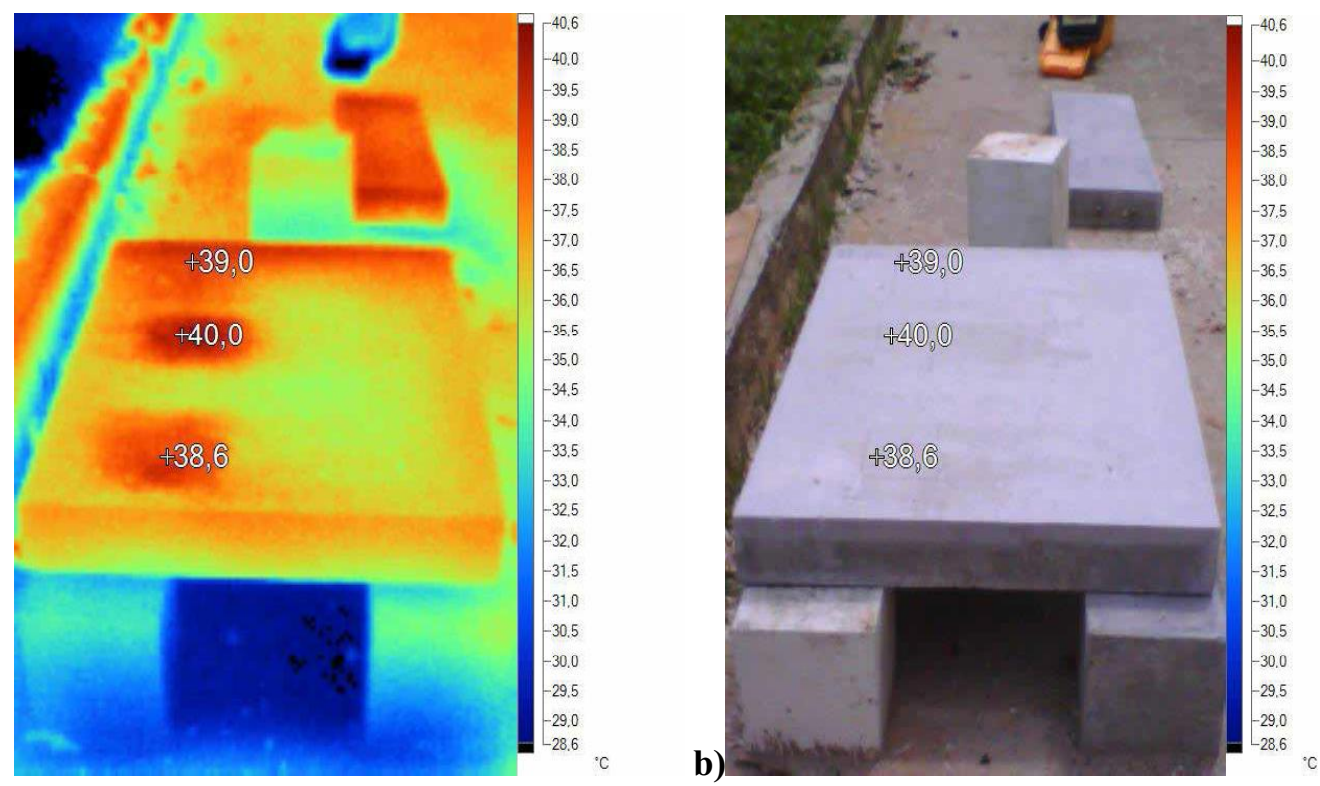

Fig. 9. a) Imagen termografía a las 11:00 a.m. B) Imagen visible a las 11:00 a.m.

En la Fig. 10 se presentan los resultados obtenidos del ensayo de martillo de rebote o esclerómetro, en comparación con los obtenidos experimentalmente en el ensayo de resistencia a la compresión de las respectivas mezclas, de cada losa.

Los resultados obtenidos de resistencia a la compresión con el martillo de rebote muestran una leve influencia por la presencia de las barras, con variación del $4.5 \%$ en la zona de la barra B1 de la losa L1. Las zonas restantes presentan un comportamiento cercano al valor experimental con diferencias menores a $2 \mathrm{MPa}$; lo anterior ocurre, probablemente, por la ubicación cercana a la superficie de la barra indicada.

Los resultados obtenidos sobre el concreto en zonas sin refuerzo para las losas L1 y L3 presentaron un aumento de $5.24 \%$ y $2.13 \%$ respecto al obtenido en el ensayo de resistencia a la compresión; la losa L2 presentó una resistencia $18.63 \%$ mayor respecto a la resistencia a la compresión experimental; esta diferencia se debió a posibles golpes en zonas con presencia de agregado grueso sub-superficial de mayor dureza o a la subjetividad propia del ensayo respecto al ángulo de ubicación del esclerómetro. En la losa L1 la diferencia del valor promedio de resistencia obtenido sobre las zonas con refuerzo y sin refuerzo es menor al $1.52 \%$. El ensayo de esclerometría se realizó a los 56 días de curado, y sus resultados se comparan con los obtenidos del ensayo de resistencia a la compresión a 28 días de curado. Debido a que el concreto alcanza la máxima resistencia a la compresión a los 28 días de curado y tiene sólo un pequeño aumento después de este tiempo, se consideró que no habría variación significativa en los resultados de resistencia estimados mediante la técnica de esclerometría a los 56 días de curado, obteniéndose un resultado muy cercano al real. 


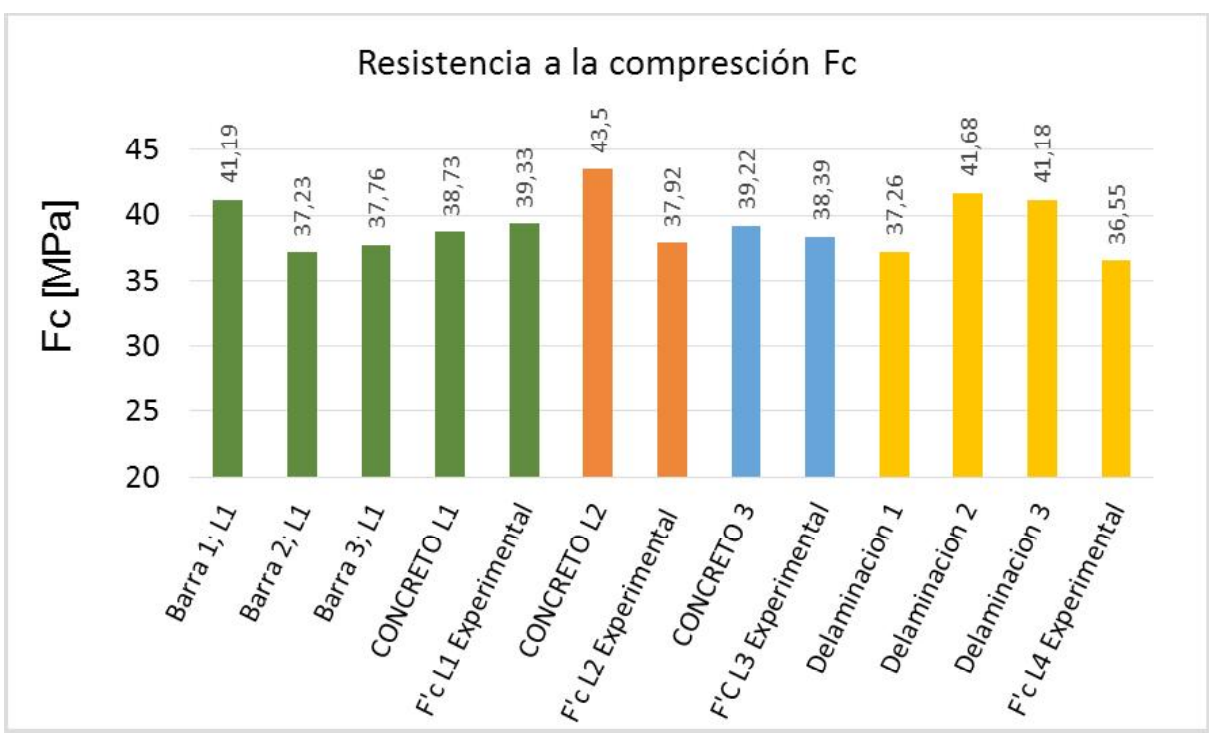

FiG. 10. Resultados del martillo de rebote medidos en L1, L2, L3 y L4.

Finalmente, en la Tabla 2 se presenta una comparación de las características ofrecidas por las técnicas no destructivas evaluadas en los elementos citados. Las tres técnicas utilizadas en la evaluación del concreto se pueden aplicar in situ, y su costo corresponde, principalmente, a la adquisición del respectivo equipo. En cuanto a la estimación de la resistencia del concreto a través de las técnicas de VPU y esclerometría, es más confiable el uso de la primera cuando se usa el método directo, pues hace una evaluación volumétrica de la muestra, mientras la segunda solo toma información superficial del material. La termografía infrarroja, según los resultados obtenidos, podría servir para hacer una selección de zonas afectas en las cuales se podrían hacer verificaciones con las técnicas complementarias citadas. En cuanto a la evaluación de los refuerzos, es necesario estudiar la capacidad y sensibilidad de otro tipo de técnicas.

TABLA 2

COMPARACIÓN DE LAS TÉCNICAS UTILIZADAS

\begin{tabular}{|l|l|l|l|l|l|l|}
\hline \multicolumn{1}{|c|}{ Criterio } & Portabilidad & $\begin{array}{c}\text { Energía } \\
\text { necesaria }\end{array}$ & \multicolumn{1}{c|}{ Sensibilidad } & Calibración & Rapidez & $\begin{array}{l}\text { Interpretación } \\
\text { de datos }\end{array}$ \\
\hline $\begin{array}{l}\text { Velocidad } \\
\text { de pulso } \\
\text { ultrasónico }\end{array}$ & SI & $\begin{array}{l}\text { Batería } \\
\text { recargable }\end{array}$ & $\begin{array}{l}\text { Buena para evaluación } \\
\text { de la matriz de concreto }\end{array}$ & $\begin{array}{l}\text { Barra de } \\
\text { calibración }\end{array}$ & Media & Medio \\
\hline $\begin{array}{l}\text { Termografía } \\
\text { Infrarroja }\end{array}$ & SI & $\begin{array}{l}\text { Batería } \\
\text { recargable }\end{array}$ & $\begin{array}{l}\text { Alta para detección de } \\
\text { delaminaciones }\end{array}$ & Automática & Rápida & Fácil \\
\hline $\begin{array}{l}\text { Martillo de } \\
\text { rebote }\end{array}$ & SI & N/A & $\begin{array}{l}\text { Buena para el estado } \\
\text { superficial de la matriz } \\
\text { de concreto }\end{array}$ & $\begin{array}{l}\text { Probeta de } \\
\text { calibración }\end{array}$ & Rápida & Fácil \\
\hline
\end{tabular}

\section{Conclusiones}

Se verificó la capacidad de la técnica de VPU para la evaluación de la homogeneidad y calidad de la matriz de concreto. Además, se comprobó el aumento de la VPU cuando el haz ultrasónico viaja paralelo al acero de refuerzo, disminuyendo a medida que el grado de daño por corrosión se hace más severo, probablemente debido al efecto de la capa de óxido sobre la velocidad de propagación de la onda. 
La baja sensibilidad de la VPU para identificar los refuerzos perpendiculares a la dirección de propagación del haz sonoro permitiría evaluar la falta de adherencia del refuerzo o la presencia de grietas en la matriz de concreto en la zona cercana al refuerzo. Además, por el método indirecto se podría estimar la profundidad de una grieta transversal al refuerzo sin tener afectación sobre las lecturas por la presencia de estos.

La termografía infrarroja presentó alta sensibilidad para captar delaminaciones simuladas en la losa de concreto, gracias al diferencial térmico generado por la concentración de calor en las zonas con presencia de delaminaciones, obteniendo una disminución en la calidad de la imagen para la delaminación ubicada a profundidad de $75 \%(7,5 \mathrm{~cm})$ del espesor de la losa. Estos resultados muestran una alternativa ágil y confiable para la inspección de elementos de concreto que estén expuestos a la radiación solar.

La técnica de martillo de rebote permitió estimar la resistencia a la compresión de la superficie del concreto, mostrando afectación en sus resultados por la presencia del agregado grueso sub-superficial de mayor dureza y por la subjetividad del ensayo.

La evaluación de las técnicas permite concluir que aunque cada técnica aporta información importante sobre la calidad o la condición del concreto o sus refuerzos, es indispensable una adecuada combinación de estas para conocer el estado global real de un puente.

\section{REFERENCIAS}

[1] L. E. Yamin y D. Ruiz, "Vulnerabilidad sísmica de puentes en Colombia y estrategias para rehabilitación", Revista de Ingeniería Universidad de los Andes, vol. 13, pp. 1-7, May. 2001.

[2] E. Muñoz, R. Hernández, "Rehabilitación de los puentes de la red de carreteras de Colombia, basados en inspecciones visuales, estudios especializados y estrategias de reparación", Revista de Ingenieria de Construcción,vol. 20, pp. 159-183, enero, 2005.

[3] I. Quashie, K. A. Danso, H.C. Odoi, C.A. Adjei et al., "Non-Destructive Evaluation of Concrete using Ultrasonic Pulse Velocity", Research Journal of Applied Sciences Engineering and Technology, vol. 3, pp.241-251, Jun. 2011.

[4] J. O. Herrera, R. Cruz y L. Quintero, "Evaluación del efecto de barras de refuerzo en concreto sobre las medidas de velocidad de pulso ultrasónico (VPU)", Revista Colombiana de Materiales, vol. 5, pp. 107-113, May. 2014.

[5] B. Milovanovic' et al., "The Role of Infrared Thermography in Nondestructive Testing of Concrete Structures", Zagreb SPIN Meeting., vol. 6, pp. 1-6, May. 2012.

[6] B. Mohammed, N. Juaini y M. Abdullahi, "Evaluation of rubbercrete based on ultrasonic pulse velocity and rebound hammer test", Construction and Building Materials., vol. 25, pp.1388-1397, Mar. 2011.

[7] G. Willian, Propuesta de una metodología no destructiva para la inspección de puentes de concreto reforzado en servicio, Bucaramanga: Universidad Industrial de Santander (UIS), 2012.

[8] L. Jones, "Inspection methods and techniques to determine Non Visible Corrosion of prestress in Strands in Concrete Bridge Components", Washington: ATLSS, 2011.

[9] Government of India, Guidelines on nondestructive testing of bridges, LUCKNOW: Research Desings and Standards Organisation, 2009.

[10] N. Zoidids, E. Tatsis, C. Vlachopoulos et al., "Inspection, evaluation and repair monitoring of cracked concrete floor using NDT methods", Construction and Building Materials, vol. 48, pp. 1302-1308, Jul. 2013.

[11] K. Harris, H. Melo et al., "Application of Thermal IR Imagery for concrete bridge inspection", CEE, vol. 36, pp. 689-704, Nov. 2011.

[12] N. Kalos, L. Sangwook y S. Hyoung, "NonDestructive Testing Methods in the U.S. for Bridge Inspection and Maintenance", KSCE Journal of Civil Engineering, pp. 1322-1331, May. 2014.

[13] R. Cruz, L. Quintero, E. Espinosa y C. Galán, "Evaluación de ensayos no destructivos para identificar deterioro en puentes de concreto", Revista Colombiana de Materiales, vol. 5, pp. 55-60, May. 2014. 
[14] Board Trasportation Research, Nondestructive testing to identify concrete bridge deck deterioration, Washington: TRB, 2012.

[15] T. Sartorti et al.,"Identificación y análisis de patologías en puentes de carreteras urbanas y rurales", Revista de Ingeniería de Construcción, vol. 26, pp. 5-24,Apr. 2011.

[16] Universidad Nacional de Colombia - Instituto Nacional de Vías (INVIAS). "Estudio e
Investigación del Estado Actual de las Obras de la Red Nacional de Carreteras. Manual para la Inspección Visual de Puentes y Pontones". Bogotá, 2006.

[17] S. Guzmán, "Modelización del deterioro de tableros de puentes de hormigón por difusión de cloruros y corrosión de la armadura pasiva", Madrid: Universidad Politécnica de Madrid, 2010. 